\title{
Bremsstrahlung of relativistic electrons in the Aharonov-Bohm potential
}

\author{
Jürgen Audretsch円 and Ulf Jasper \\ Fakultät für Physik der Universität Konstanz, Postfach 5560, D 78434 Konstanz, Germany \\ Vladimir D. Skarzhinsky \\ Fakultät für Physik der Universität Konstanz, Postfach 5560, D 78434 Konstanz, Germany \\ and P. N. Lebedev Physical Institute, Leninsky prospect 53, Moscow 117924, Russia
}

(Phys.Rev.,D, 53, 2178, 1996)

\begin{abstract}
We investigate the scattering of an electron by an infinitely thin and infinitely long straight magnetic flux tube in the framework of QED. We discuss the solutions of the Dirac and Maxwell fields in the related external pure $\mathrm{AB}$ potential and evaluate matrix elements and differential probabilities for the bremsstrahlung process. The dependence of the resulting cross section on the energy, direction and polarization of the involved particles is analyzed. In the low energy regime a surprising angular asymmetry is found which results from the interaction of the electron's magnetic moment with the magnetic field.

PACS numbers: 03.65.Bz, 12.20.-m
\end{abstract}

\section{Introduction}

In classical physics the behaviour of charged particles in external electro-magnetic fields is completely determined by the electric and magnetic field strengths, $F_{\mu \nu}=\partial_{\mu} A_{\nu}-\partial_{\nu} A_{\mu}$, which fix their trajectories by way of the resulting forces. For quantum particles, however, the concept of field strengths, which are gauge invariant local quantities, is insufficient. This became evident with the prediction of the AharonovBohm (AB) effect [1] (see also [2, 3|), which states the influence of magnetic fluxes on quantum systems. It was shown that it is the line integrals of the vector potential over closed paths which produce the additional effects on quantum systems [ $\left[\right.$. The path-dependent phase factors $\exp \left(i e \oint A_{\mu} d x_{\mu}\right)$, which form a class of topological, and again, gauge invariant quantities, produce phase shifts in wave functions. A number of remarkable experiments was made to observe the resulting interference pattern of an electron beam scattered by a thin solenoid. For a comprehensive review see $[5$, 6. In solid state physics the manifestation of the AB-effect brought new unexpected results [7, 8]. The influence of magnetic fluxes on vacuum polarization of quantum fields was discussed in [9]. For a detailed investigation of the $\mathrm{AB}$ effect and its physical consequences it is essential not only to consider the scattering but also accompanying effects. Among them bremsstrahlung is supposed to be the most significant one. It was discussed at first for non-relativistic particles in the dipole approximation in [10], where the energetic and angular distributions were calculated. The polarization properties of the bremsstrahlung were discussed for relativistic spinless particles in 11. The aim of the present paper is a detailed investigation of the bremsstrahlung emitted by a Dirac electron being scattered in the AB potential. This process is evidently of greatest interest in view of a possible experimental verification using polarizable electron beams.

As usual we will consider the idealized case of an infinitely thin, infinitely long straight solenoid (magnetic string), lying along the $z$ axis. The vector potential connected with this pure $A B$ configuration reads

\footnotetext{
${ }^{1}$ e-mail: Juergen.Audretsch@uni-konstanz.de

2 e-mail: Ulf.Jasper@uni-konstanz.de

${ }^{3}$ e-mail: vdskarzh@sgi.lpi.msk.su
}

$$
\vec{A}=\frac{\Phi}{2 \pi \rho} \vec{e}_{\varphi},
$$


where $\Phi$ is the magnetic flux and $\rho$ the distance to the string. This idealization raises the question of how to describe the electron wave function near the string correctly. Mathematically this is related to the non-selfadjointness of the Hamilton operator [12] - [15]. We will discuss this point in section 2, where we investigate the Dirac equation in the presence of the pure $\mathrm{AB}$ potential. There we also find the exact scattering solutions in terms of partial waves. The issue of spin changes slightly the interpretation of the $\mathrm{AB}$ effect. As we will show the interaction between spin and magnetic field leads to the Dirac wave functions which do not vanish on the magnetic string and thus, in a way, a local element is added to the non-locality of the $\mathrm{AB}$ effect.

In section 3 the matrix element for the bremsstrahlung process is calculated and the differential radiative cross section is obtained. We analyze the behaviour of the differential and total cross section at different energy regimes and discuss its particular features characteristic for the Dirac electron in sections 4 and 5 .

We use units such that $\hbar=c=1$ and take $e<0$ for the electron charge.

\section{Solutions of the Dirac and Maxwell field in the Aharonov- Bohm potential}

The Dirac equation in an external magnetic field reads

$$
i \partial_{t} \psi=H \psi, \quad H=\alpha_{i}\left(p_{i}-e A_{i}\right)+\beta M
$$

where $e$ is the electron charge. For the matrices $\alpha$ and $\beta$ we use

$$
\alpha_{i}=\left(\begin{array}{cc}
0 & \sigma_{i} \\
\sigma_{i} & 0
\end{array}\right), \quad \beta=\left(\begin{array}{cc}
1 & 0 \\
0 & -1
\end{array}\right) .
$$

In cylindrical coordinates $(\rho, \varphi, z)$, the kinetic momenta are given by

$$
\pi_{\rho}=p_{\rho}=-i \partial_{\rho}, \quad \pi_{\varphi}=p_{\varphi}-e A_{\varphi}=-\frac{i}{\rho} \partial_{\varphi}-e A_{\varphi}, \quad p_{3}=-i \partial_{z}
$$

and

$$
\sigma_{\rho}=\sigma_{1} \cos \varphi+\sigma_{2} \sin \varphi, \quad \sigma_{\varphi}=\left(-\sigma_{1} \sin \varphi+\sigma_{2} \cos \varphi\right)
$$

where $\sigma_{i}$ are the Pauli matrices.

The vector potential for the pure $A B$ case (magnetic string) has a nonzero angular component [1]

$$
e A_{\varphi}=\frac{e \Phi}{2 \pi \rho}=-\frac{\Phi}{\Phi_{0} \rho}=\frac{\phi}{\rho},
$$

$(0 \leq \rho<\infty)$, where $\Phi$ is magnetic flux and $\Phi_{0}=2 \pi /|e|$ is the magnetic flux quantum. It corresponds to a magnetic field along the $z$ axis

$$
B_{z}=\frac{2 \phi}{e \rho} \delta(\rho),
$$

which points to the positive (negative) $z$ direction for $\phi<0(\phi>0)$. It is the fractional part $\delta$ of the magnetic flux $\phi=N+\delta, 0<\delta<1$ which produces all physical effects. Its integral part $N$ will appear as a phase factor $\exp (i N \varphi)$ in the solutions to the Dirac equation.

The exact solution of the Dirac equation used for the discussion of the scattering problem in the external Aharonov-Bohm field can be written in an integral form as it was done for the Schrödinger equation in the original paper by Y. Aharonov and D. Bohm [1]. For further calculations, however, cylindrical modes are more convenient.

For the Dirac equation in the AB field the complete set of commuting operators is

$$
\hat{H}, \quad \hat{p_{3}}:=-i \partial_{z}, \quad \hat{J}_{3}:=-i \partial_{\varphi}+\frac{1}{2} \Sigma_{3}, \quad \hat{S}_{3}:=\beta \Sigma_{3}+\gamma \frac{p_{3}}{M}
$$


where $\gamma:=\left(\begin{array}{ll}0 & 1 \\ 1 & 0\end{array}\right)$. The corresponding eigenvalue equations are given by

$$
\begin{aligned}
\hat{H} \psi & =E \psi, \\
\hat{p_{3}} \psi & =p_{3} \psi, \\
\hat{J}_{3} \psi & =j_{3} \psi, \\
\hat{S_{3}} \psi & =s \psi,
\end{aligned}
$$

where $E=\sqrt{p_{\perp}^{2}+p_{3}^{2}+M^{2}}$ is the energy, $p_{3}$ and $j_{3}$ are the $z$-components of linear and total angular momentum respectively; $p_{\perp}$ denotes the radial momentum. The eigenvalue of $\hat{J}_{3}$ is half-integer and we rewrite it by introducing $l, j_{3}=: l+N+1 / 2$. Here $l$ is an integer number and $\mathrm{N}$ is fixed as above. Note that $l+N$ denotes an integral part of the eigenvalue of $\hat{J}_{3}$ in contrast to the usual convention. The corresponding separation of a factor $\exp (i N \varphi)$ in the solutions of the Dirac equation will turn out to be convenient in the following calculations. We introduced in eq. (9) the operator $\hat{S}_{3}$ and not the helicity operator $\hat{S}_{t}=\Sigma_{i}\left(p_{i}-e A_{i}\right) / p$ which is often used. Both of these operators commute in the relativistic case with the operators $\hat{H}, \hat{p}_{3}$ and $\hat{J}_{3}$, when a magnetic field of a fixed direction is present. This can be seen, for example, in [18]. We prefer to use $\hat{S}_{3}$ because in the nonrelativistic limit, which will be treated below, it describes the spin projection along the direction of the magnetic field. Its eigenvalue is given by $s= \pm \sqrt{1+p_{3}^{2} / M^{2}}$. Solving these eigenvalue equations leads to a radial solution of Bessel type.

As independent solutions we choose Bessel functions of the first kind with positive and negative orders. Then the normalization condition for the partial modes with quantum numbers $j=\left(p_{\perp}, p_{3}, l, s\right)$,

$$
\int d x \psi^{\dagger}(j, x) \psi\left(j^{\prime}, x\right)=\delta_{j, j^{\prime}}=\delta_{s, s^{\prime}} \delta_{l, l^{\prime}} \delta\left(p_{3}-p_{3^{\prime}}\right) \frac{\delta\left(p_{\perp}-p_{\perp}^{\prime}\right)}{\sqrt{p_{\perp} p_{\perp}^{\prime}}}
$$

fixes the solutions (for electron states with $E>0$ ) only for values of $l$ outside the interval $-1<l-\delta<0$. Unless $l=0$ the Bessel functions of negative order are not square integrable and therefore the normalized solutions contain only the regular Bessel functions of positive order. One finds, for $l \neq 0$, for the total mode function

$$
\psi_{e}(j, x)=\frac{1}{2 \pi} N_{e} e^{-i E_{p} t+i p_{3} z} e^{i N \varphi} e^{i \frac{\pi}{2}|l|}\left(\begin{array}{c}
u \\
v
\end{array}\right),
$$

where

$$
\begin{aligned}
& u=\frac{1}{\sqrt{2 s}}\left(\begin{array}{c}
\sqrt{E_{p}+s M} \sqrt{s+1} J_{\nu_{1}}\left(p_{\perp} \rho\right) e^{i l \varphi} \\
i \epsilon_{3} \epsilon_{l} \sqrt{E_{p}-s M} \sqrt{s-1} J_{\nu_{2}}\left(p_{\perp} \rho\right) e^{i(l+1) \varphi}
\end{array}\right), \\
& v=\frac{1}{\sqrt{2 s}}\left(\begin{array}{c}
\epsilon_{3} \sqrt{E_{p}+s M} \sqrt{s-1} J_{\nu_{1}}\left(p_{\perp} \rho\right) e^{i l \varphi} \\
i \epsilon_{l} \sqrt{E_{p}-s M} \sqrt{s+1} J_{\nu_{2}}\left(p_{\perp} \rho\right) e^{i(l+1) \varphi}
\end{array}\right),
\end{aligned}
$$

and

$$
\begin{gathered}
N_{e}:=\frac{1}{\sqrt{2 E_{p}}}, \quad p_{\perp}:=\sqrt{p^{2}-p_{3}^{2}}=\sqrt{E_{p}^{2}-M^{2}-p_{3}^{2}}, \quad s= \pm \sqrt{1+\frac{p_{3}^{2}}{M^{2}}}, \quad \epsilon_{3}:=\operatorname{sign}\left(s p_{3}\right), \\
\nu_{1}:=\left\{\begin{array}{r}
l-\delta \\
-l+\delta
\end{array}, \quad \nu_{2}:=\left\{\begin{array}{r}
l+1-\delta \\
-l-1+\delta
\end{array}, \quad \epsilon_{l}:=\left\{\begin{array}{rl}
1 & \text { if } l \geq 0 \\
-1 & \text { if } l<0
\end{array} .\right.\right.\right.
\end{gathered}
$$

In (15) we already included without proof the case $l=0$. This critical mode, however, requires a separate discussion, to which we will turn now.

The solution for $l=0$ contains Bessel functions of positive and negative orders. It can be written as

$$
\begin{aligned}
u & =\frac{N_{0}}{\sqrt{2 s}}\left(\begin{array}{c}
\sqrt{E_{p}+s M} \sqrt{s+1}\left[\sin \mu J_{-\delta}\left(p_{\perp} \rho\right)+\cos \mu J_{\delta}\left(p_{\perp} \rho\right)\right] \\
i \epsilon_{3} \sqrt{E_{p}-s M} \sqrt{s-1}\left[\sin \mu J_{1-\delta}\left(p_{\perp} \rho\right)-\cos \mu J_{-1+\delta}\left(p_{\perp} \rho\right)\right] e^{i \varphi}
\end{array}\right), \\
v & =\frac{N_{0}}{\sqrt{2 s}}\left(\begin{array}{c}
\epsilon_{3} \sqrt{E_{p}+s M} \sqrt{s-1}\left[\sin \mu J_{-\delta}\left(p_{\perp} \rho\right)+\cos \mu J_{\delta}\left(p_{\perp} \rho\right)\right] \\
i \sqrt{E_{p}-s M} \sqrt{s+1}\left[\sin \mu J_{1-\delta}\left(p_{\perp} \rho\right)-\cos \mu J_{-1+\delta}\left(p_{\perp} \rho\right)\right] e^{i \varphi}
\end{array}\right)
\end{aligned}
$$

where $\mu$ is an arbitrary parameter. It is normalizable for any value of $\mu$. Moreover one can see that there is no solution that behaves regular at $\rho=0$ as it is the case for the spinless case. At least one component 
of each two-spinor diverges at $\rho=0$. This is an obvious consequence of the additional interaction between the magnetic moment of the electron and magnetic field inside the solenoid. The question remains, what value of $\mu$ is to be chosen?

Note that the first spinor component is square integrable but singular for $l=0$ as well as for $l=1$. (One has to replace $\delta$ with $-l+\delta$ in eq. (16) in order to get the general solution.) But for $l=1$ the second component turns out to be not square integrable. This is the reason why only one critical mode appears, in contrast to [13.

The zero mode problem is not specific for the Dirac equation. It is related to the fact that the Hamilton operator is not selfadjoint in the presence of the pure Aharonov-Bohm potential for any charged quantum system. This would cause many problems for the unitary evolution of quantum systems unless selfadjoint extensions of these Hamilton operators exist. The related self-adjoint extension procedure [16] permits to fix the parameter $\mu$ up to an arbitrary constant. We shall not describe this method here. In 15 we presented an alternative method of treating the self-adjointness problem. It essentially amounts to finding orthogonal states with respect to the radial momentum $p_{\perp}$ for the critical mode $(l=0)$. It then immediately leads to the same condition on the parameter $\mu$ as the self-adjoint extension method does:

$$
\tan \mu=\alpha\left(\frac{p_{\perp}}{M}\right)^{2 \delta} \frac{M}{E_{p}+s M}, \quad \alpha=\text { const }, \quad N_{0}=\frac{1}{\sqrt{1+\sin 2 \mu \cos \pi \delta}} .
$$

If these conditions are fulfilled, then the cylindrical modes to the Dirac equation form a complete set of functions for any $\alpha$. Similar results can be obtained for other quantum systems in the AharonovBohm potential both relativistic and nonrelativistic ones. Note that the self-adjoint extension method does not fix the open parameter $\alpha$ which determines the behaviour of the wave function at the origin. This situation is not satisfactory from the physical point of view (in addition the calculations in the following sections cannot be performed for arbitrary $\alpha$ ). We solve this problem as follows: The pure Aharonov-Bohm potential is the limiting case of nonsingular vector potentials of real solenoids of finite radii. Hamiltonians in the presence of realistic magnetic fields are selfadjoint operators, and one would expect that the limiting procedure permits us to fix the arbitrary parameter $\alpha$ completely. This was shown in the paper 12] in general and we will confirm this result for the special case of a cylindrical solenoid with uniform magnetic field inside. The vector potential outside the solenoid coincides with (3). Inside it depends on the distribution of the magnetic field. We choose a uniform distribution, so that

$$
e A_{\varphi}=\frac{\phi \rho}{a^{2}}, \quad \text { if } \quad \rho<\mathrm{a},
$$

where $a$ is the radius of the solenoid with the magnetic field $H=2 \phi / e a^{2}$.

Solutions to the Dirac equation contain the Bessel functions with positive and negative orders for $\rho>a$ and a confluent hypergeometric function for $\rho<a$. The matching and normalization conditions will fix all arbitrary coefficients in these solutions. It is evident that for $l \neq 0$ the pure AB solution will be restored in the limiting case of vanishing radius. For $l=0$ one can expect that this procedure will lead to a self-adjoint extension of the pure AB Dirac operator. So we need to consider only this special case.

The equations for radial functions are

$$
R_{1,2}^{\prime \prime}+\frac{1}{\rho} R_{1,2}^{\prime}-\frac{1}{\rho^{2}}\left(l_{1,2}-\frac{\phi \rho^{2}}{a^{2}}\right)^{2} R_{1,2}+p_{1,2}^{2} R_{1,2}=0
$$

with

$$
l_{1,2}=\left(\begin{array}{c}
N \\
N+1
\end{array}\right), \quad p_{1,2}^{2}=p_{\perp}^{2} \pm \frac{2 \phi}{a^{2}} .
$$

Solutions to these equations that are regular at $\rho=0$ are

$$
R_{1,2}=x^{\frac{l_{1,2}}{2}} e^{-\frac{x}{2}} \Phi\left(A_{1,2}, C_{1,2} ; x\right)
$$

where $\Phi(A, C ; x)$ is the confluent hypergeometric function with

$$
x=|\phi| \frac{\rho^{2}}{a^{2}}, \quad A_{1,2}=-\frac{p_{1,2}^{2} a^{2}}{4|\phi|}+\frac{1}{2}, \quad C_{1,2}=l_{1,2}+\frac{1}{2} .
$$


One can see from the matching conditions that external solution will not contain the Bessel function with a negative order unless the parameter $A_{1,2}$ goes to zero at $a \rightarrow 0$. It happens for the $R_{1}$ component at $\phi>0$ when the magnetic field directs down and for $R_{2}$ component at $\phi<0$. In these cases the interaction of the electron magnetic moment with the magnetic field is attractive and the probability to find the electron near the string increases.

Thus, at $N>0$ when the magnetic field points to the negative $z$ direction, which we shall assume from now on, the upper two-spinor component (corresponding to spin up) is enhanced and one needs to put the parameter $\mu$ in the equation (18) and the solution (16) equal to $\pi / 2$. The self-adjoint extension parameter becomes $\alpha=\infty$. Then each component of the zero mode solution (16) contains only one Bessel function and therefore we can include the $l=0$ case in (15), what was to be shown. Eqn.(11) with (12) - 15) represents the central result of this section.

Let us remark that the method of taking the zero radius limit of a finite flux tube, as sketched above, does not give physically meaningful results if an additional Coulomb potential is present [20]. However, we do not consider that case here.

The expression (11) with (12) and (13) presents the partial electron wave functions in terms of cylindrical modes. The electron scattering wave function is obtained by a superposition

$$
\Psi(\vec{p}, s, x):=\sum_{l} c_{l} \psi_{e}(j, x)
$$

With the coefficients

$$
c_{l}^{(+)}:=e^{-i l \varphi_{p}} e^{i \frac{\pi}{2} \epsilon_{l} \delta}
$$

this solution behaves at large distances like a plane wave, propagating in the direction of $\vec{p}$ given by $p_{x}=p_{\perp} \cos \varphi_{p}, p_{y}=p_{\perp} \sin \varphi_{p}, p_{z}=p_{3}$, plus an outgoing cylindrical wave,

$$
\Psi(\vec{p}, s, x) \sim\left(e^{i \vec{p} \vec{x}}+f(\varphi) \sqrt{\frac{i}{\rho}} e^{i p_{\perp} \rho+i p_{3} z}\right) e^{-i \omega t}
$$

Because of the damping of the cylindrical wave at large distances we may use this superposition instead of plane waves. Accordingly, the coefficients

$$
c_{l}^{(-)}:=e^{-i l \varphi_{p}} e^{-i \frac{\pi}{2} \epsilon_{l} \delta}
$$

are used to form a scattering wave solution for an outgoing electron with an ingoing cylindrical wave. With this different choice of the coefficients for ingoing and outgoing electrons the interaction with the external AB-field will be described correctly.

The external Aharonov-Bohm field does not influence the photon wave function. In cylindrical coordinates it reads

$$
A_{\mu}^{\lambda}(\vec{k}, x)=\frac{e_{\mu}^{(\lambda)}}{\sqrt{2 \omega_{k}}} e^{-i \omega_{k} t+i k_{3} z} e^{i k_{\perp} \rho \cos \left(\varphi-\varphi_{k}\right)}
$$

where the polarization vectors for two physical transversal photons (in cartesian coordinates)

$$
e^{(\sigma)}:=\left(0,-\sin \varphi_{k}, \cos \varphi_{k}, 0\right), \quad e^{(\pi)}:=\frac{1}{\omega_{k}}\left(0,-k_{3} \cos \varphi_{k},-k_{3} \sin \varphi_{k}, k_{\perp}\right)
$$

correspond to two linear polarization states.

\section{Matrix elements and differential cross sections for the process $e \rightarrow e+\gamma$}

Cross sections are usually related to plane wave states, and in our case to the scattering states (22). The cylindrical modes (11) have a vanishing radial flux and therefore do not describe ingoing or outgoing particles. They are, however, convenient for calculating matrix elements and we shall use these matrix elements as starting point for the calculation of the cross section which refers to scattering states. 


\subsection{Matrix elements for cylindrical modes}

The matrix element for the bremsstrahlung process for an ingoing electron with quantum numbers $j_{p}=\left(p_{\perp}, p_{3}, l, s\right)$ leading to an outgoing electron with quantum numbers $j_{q}=\left(q_{\perp}, q_{3}, n, r\right)$ and a photon with quantum numbers $j_{k}=(\vec{k}, \lambda)$ for physical states $\lambda=(\sigma, \pi)$ has the usual form

$$
\widetilde{M}_{\lambda}\left(j_{p}, j_{q}\right)=-i\left\langle j_{q}, j_{k}\left|S^{(1)}\right| j_{p}\right\rangle=-e \int d^{4} x \bar{\psi}_{e}\left(j_{q}, x\right) A_{\mu}^{* \lambda}(\vec{k}, x) \gamma^{\mu} \psi_{e}\left(j_{p}, x\right)
$$

whereby gamma matrices are written in terms of Pauli matrices as

$$
\gamma_{i}=\left(\begin{array}{cc}
0 & \sigma_{i} \\
-\sigma_{i} & 0
\end{array}\right)
$$

so that

$$
e_{\mu}^{*(\lambda)} \gamma_{\mu}=\left(\begin{array}{cc}
0 & \alpha_{\lambda} \\
-\alpha_{\lambda} & 0
\end{array}\right), \alpha_{\sigma}=\left(\begin{array}{cc}
0 & -i e^{-i \varphi_{k}} \\
i e^{i \varphi_{k}} & 0
\end{array}\right), \alpha_{\pi}=\left(\begin{array}{cc}
k_{\perp} & -k_{3} e^{-i \varphi_{k}} \\
-k_{3} e^{i \varphi_{k}} & -k_{\perp}
\end{array}\right) \frac{1}{\omega_{k}} .
$$

Using the expressions (11), (12), (13) and (25), we can rewrite the matrix element (27) in the form

$$
\widetilde{M}_{\lambda}\left(j_{p}, j_{q}\right)=-e \frac{1}{2 \sqrt{2 \omega_{k} E_{q} E_{p}}} e^{\left(i \frac{\pi}{2}(|l|-|n|)\right.} \delta\left(E_{p}-E_{q}-\omega_{k}\right) \delta\left(p_{3}-q_{3}-k_{3}\right) m_{\lambda},
$$

with

$$
\begin{aligned}
m_{\lambda} & :=\int \rho d \rho d \varphi e^{-i k_{\perp} \rho \cos \left(\varphi-\varphi_{k}\right)}\left[u^{\dagger}(q) \alpha_{\lambda} v(p)+v^{\dagger}(q) \alpha_{\lambda} u(p)\right] \\
& =e^{i(l-n) \varphi_{k}} \int \rho d \rho d \varphi e^{-i k_{\perp} \rho \cos \left(\varphi-\varphi_{k}\right)} K_{\lambda}(\rho, \varphi) .
\end{aligned}
$$

For the polarization state $\lambda=\sigma$ we have

$$
\begin{aligned}
K_{\sigma}(\rho, \varphi)= & R\left[\epsilon_{l} \sqrt{E_{q}+r M} \sqrt{E_{p}-s M} J_{\nu_{1}}\left(q_{\perp} \rho\right) J_{\nu_{2}}\left(p_{\perp} \rho\right) e^{i(l-n+1)\left(\varphi-\varphi_{k}\right)}\right. \\
& \left.+\epsilon_{n} \sqrt{E_{q}-r M} \sqrt{E_{p}+s M} J_{\nu_{2}}\left(q_{\perp} \rho\right) J_{\nu_{1}}\left(p_{\perp} \rho\right) e^{i(l-n-1)\left(\varphi-\varphi_{k}\right)}\right]
\end{aligned}
$$

and for the polarization state $\lambda=\pi$

$$
\begin{aligned}
K_{\pi}(\rho, \varphi)= & \frac{k_{\perp}}{\omega_{k}} R_{\perp}\left[\sqrt{E_{q}+r M} \sqrt{E_{p}+s M} J_{\nu_{1}}\left(q_{\perp} \rho\right) J_{\nu_{1}}\left(p_{\perp} \rho\right)\right. \\
& \left.-\epsilon_{n} \epsilon_{l} \sqrt{E_{q}-r M} \sqrt{E_{p}-s M} J_{\nu_{2}}\left(q_{\perp} \rho\right) J_{\nu_{2}}\left(p_{\perp} \rho\right)\right] e^{i(l-n)\left(\varphi-\varphi_{k}\right)} \\
& -\frac{i k_{3}}{\omega_{k}} R\left[\epsilon_{l} \sqrt{E_{q}+r M} \sqrt{E_{p}-s M} J_{\nu_{1}}\left(q_{\perp} \rho\right) J_{\nu_{2}}\left(p_{\perp} \rho\right) e^{i(l-n+1)\left(\varphi-\varphi_{k}\right)}\right. \\
& \left.-\epsilon_{n} \sqrt{E_{q}-r M} \sqrt{E_{p}+s M} J_{\nu_{2}}\left(q_{\perp} \rho\right) J_{\nu_{1}}\left(p_{\perp} \rho\right) e^{i(l-n-1)\left(\varphi-\varphi_{k}\right)}\right]
\end{aligned}
$$

where

$$
\begin{aligned}
R & :=\frac{\sqrt{r+1} \sqrt{s+1}+\epsilon_{3}(q) \epsilon_{3}(p) \sqrt{r-1} \sqrt{s-1}}{2 \sqrt{s r}}, \\
R_{\perp} & :=\frac{\epsilon_{3}(q) \sqrt{r-1} \sqrt{s+1}+\epsilon_{3}(p) \sqrt{r+1} \sqrt{s-1}}{2 \sqrt{s r}} .
\end{aligned}
$$

Integrating over $\varphi$ we obtain

$$
\begin{aligned}
m_{\sigma}= & 2 \pi e^{i(l-n) \varphi_{k}} R \\
& \times\left[\epsilon_{l} \sqrt{E_{q}+r M} \sqrt{E_{p}-s M} e^{-i \frac{\pi}{2}(l-n+1)} \int \rho d \rho J_{\nu_{1}}\left(q_{\perp} \rho\right) J_{\nu_{2}}\left(p_{\perp} \rho\right) J_{l-n+1}\left(k_{\perp} \rho\right)\right. \\
& \left.+\epsilon_{n} \sqrt{E_{q}-r M} \sqrt{E_{p}+s M} e^{-i \frac{\pi}{2}(l-n-1)} \int \rho d \rho J_{\nu_{2}}\left(q_{\perp} \rho\right) J_{\nu_{1}}\left(p_{\perp} \rho\right) J_{l-n-1}\left(k_{\perp} \rho\right)\right] .
\end{aligned}
$$


and

$$
\begin{array}{rl}
m_{\pi}=2 & 2 \pi e^{i(l-n) \varphi_{k}} e^{-i \frac{\pi}{2}(l-n)} \\
\times & \left\{\frac { k _ { \perp } } { \omega _ { k } } R _ { \perp } \left[\sqrt{E_{q}+r M} \sqrt{E_{p}+s M} \int \rho d \rho J_{\nu_{1}}\left(q_{\perp} \rho\right) J_{\nu_{1}}\left(p_{\perp} \rho\right) J_{l-n}\left(k_{\perp} \rho\right)\right.\right. \\
& \left.-\epsilon_{n} \epsilon_{l} \sqrt{E_{q}-r M} \sqrt{E_{p}-s M} \int \rho d \rho J_{\nu_{2}}\left(q_{\perp} \rho\right) J_{\nu_{2}}\left(p_{\perp} \rho\right) J_{l-n}\left(k_{\perp} \rho\right)\right] \\
- & \frac{k_{3}}{\omega_{k}} R\left[\epsilon_{l} \sqrt{E_{q}+r M} \sqrt{E_{p}-s M} \int \rho d \rho J_{\nu_{1}}\left(q_{\perp} \rho\right) J_{\nu_{2}}\left(p_{\perp} \rho\right) J_{l-n+1}\left(k_{\perp} \rho\right)\right. \\
& \left.\left.+\epsilon_{n} \sqrt{E_{q}-r M} \sqrt{E_{p}+s M} \int \rho d \rho J_{\nu_{2}}\left(q_{\perp} \rho\right) J_{\nu_{1}}\left(p_{\perp} \rho\right) J_{l-n-1}\left(k_{\perp} \rho\right)\right]\right\} .
\end{array}
$$

It follows from the energy conservation $\delta$ term in $(30$ ) that the radial momenta of the particles fulfill $p_{\perp}>q_{\perp}+k_{\perp}$. The excess of radial momentum, $\Delta=1-\left(q_{\perp}+k_{\perp}\right) / p_{\perp}$, is transmitted to the flux tube. For this case, using formulae [6.578(3), 6.522(14)] of [17], one can see that the integrals over $\rho$ vanish unless $\left(l+\frac{1}{2}\right)\left(n+\frac{1}{2}\right)<0$. This inequality is fulfilled at $l \geq 0, n<0$ and $n \geq 0, l<0$, and the nonvanishing integrals are of the type

$$
\begin{aligned}
J(\alpha, \beta) & :=\int_{0}^{\infty} \rho d \rho J_{\alpha}\left(p_{\perp} \rho \sin A \cos B\right) J_{\beta}\left(p_{\perp} \rho \cos A \sin B\right) J_{\beta-\alpha}\left(p_{\perp} \rho\right) \\
& =\frac{2 \sin \pi \alpha}{\pi p_{\perp}^{2} \cos (A+B) \cos (A-B)}\left(\frac{\sin A}{\cos B}\right)^{\alpha}\left(\frac{\sin B}{\cos A}\right)^{\beta}
\end{aligned}
$$

with $q_{\perp}=p_{\perp} \sin A \cos B, k_{\perp}=p_{\perp} \sin B \cos A$.

The partial wave analysis of the bremsstrahlung process in the Abahronov-Bohm potential shows a rather unexpected feature: The process turns out to be forbidden unless the quantum numbers $l$ and $n$ of the ingoing and outgoing electron have opposite signs. This in turn implies that their kinetic angular momentum projections, $[\vec{r} \times(\vec{p}-e \vec{A})]_{3}=-i \partial_{\varphi}-\phi$, have opposite signs. To see this one calculates the corresponding expectation values and finds

$$
\begin{aligned}
\left\langle[\vec{r} \times(\vec{p}-e \vec{A})]_{3}\right\rangle & =\left\langle-i \partial_{\varphi}-\phi\right\rangle \\
& =l+\frac{1}{2}-\delta-\frac{1}{2} s \frac{M}{E_{p}}=l-\delta+\frac{1}{2}\left(1 \pm \frac{\sqrt{M^{2}+p_{3}^{2}}}{\sqrt{M^{2}+p_{3}^{2}+p_{\perp}^{2}}}\right),
\end{aligned}
$$

from which it follows that the sign of $l$ equals the sign of $\left\langle[\vec{r} \times(\vec{p}-e \vec{A})]_{3}\right\rangle$ for all values of $\delta$, s, and $l \neq 0$. In the framework of a semiclassical picture this leads to the interpretation that the electrons need to pass the magnetic string in opposite directions. Apparently this is necessary for the ingoing electron to give the excess of its radial momentum to the string and to emit a real bremsstrahlung photon.

Using expression (38) we find for the matrix element (36)

$$
\begin{aligned}
m_{\sigma}= & -2 \pi i e^{i(l-n) \varphi_{k}} e^{-i \frac{\pi}{2}|l-n|} R \\
\times & \left\{\Theta ( l \geq 0 ) \Theta ( n < 0 ) \left[\sqrt{E_{q}+r M} \sqrt{E_{p}-s M} J(-n+\delta, l-n+1)\right.\right. \\
& \left.+\sqrt{E_{q}-r M} \sqrt{E_{p}+s M} J(-n-1+\delta, l-n-1)\right] \\
+ & \Theta(l<0) \Theta(n \geq 0)\left[\sqrt{E_{q}+r M} \sqrt{E_{p}-s M} J(n-\delta,-l+n-1)\right. \\
& \left.\left.+\sqrt{E_{q}-r M} \sqrt{E_{p}+s M} J(n+1-\delta,-l+n+1)\right]\right\} \\
=- & \frac{4 i R e^{i(l-n) \varphi_{k}-i \frac{\pi}{2}|l-n|+i \pi n} \sin \pi \delta}{\sqrt{p_{\perp}^{4}-2 p_{\perp}^{2}\left(q_{\perp}^{2}+k_{\perp}^{2}\right)+\left(q_{\perp}^{2}-k_{\perp}^{2}\right)^{2}}(a b)^{|n|} b^{|l|}} \\
\times & \left\{\sqrt{E_{q}+r M} \sqrt{E_{p}-s M}\left[\Theta(l \geq 0) \Theta(n<0) a^{\delta} b-\Theta(l<0) \Theta(n \geq 0) a^{-\delta} b^{-1}\right]\right. \\
- & \left.\sqrt{E_{q}-r M} \sqrt{E_{p}+s M}\left[\Theta(l \geq 0) \Theta(n<0) a^{-1+\delta} b^{-1}-\Theta(l<0) \Theta(n \geq 0) a^{1-\delta} b\right]\right\},
\end{aligned}
$$


where we have introduced

$$
\begin{aligned}
a & :=\frac{\sin A}{\cos B}=\frac{p_{\perp}^{2}-k_{\perp}^{2}+q_{\perp}^{2}-\sqrt{p_{\perp}^{4}-2 p_{\perp}^{2}\left(q_{\perp}^{2}+k_{\perp}^{2}\right)+\left(q_{\perp}^{2}-k_{\perp}^{2}\right)^{2}}}{2 p_{\perp} q_{\perp}}, \\
b & :=\frac{\sin B}{\cos A}=\frac{p_{\perp}^{2}+k_{\perp}^{2}-q_{\perp}^{2}-\sqrt{p_{\perp}^{4}-2 p_{\perp}^{2}\left(q_{\perp}^{2}+k_{\perp}^{2}\right)+\left(q_{\perp}^{2}-k_{\perp}^{2}\right)^{2}}}{2 p_{\perp} q_{\perp}} .
\end{aligned}
$$

In the same way we find for the matrix element (37)

$$
m_{\pi}=2 \pi e^{i(l-n) \varphi_{k}} e^{-i \frac{\pi}{2}|l-n|}[\Theta(l \geq 0) \Theta(n<0) L+\Theta(l<0) \Theta(n \geq 0) N] \frac{1}{\omega_{k}},
$$

with

$$
\begin{aligned}
L:=\quad k_{\perp} & R_{\perp}\left[\sqrt{E_{q}+r M} \sqrt{E_{p}+s M} J(-n+\delta, l-n)\right. \\
& \left.+\sqrt{E_{q}-r M} \sqrt{E_{p}-s M} J(-n-1+\delta, l-n)\right] \\
- & k_{3} R\left[\sqrt{E_{q}+r M} \sqrt{E_{p}-s M} J(-n+\delta, l-n+1)\right. \\
- & \left.\sqrt{E_{q}-r M} \sqrt{E_{p}+s M} J(-n-1+\delta, l-n-1)\right],
\end{aligned}
$$

and

$$
\begin{aligned}
N:=\quad k_{\perp} & R_{\perp}\left[\sqrt{E_{q}+r M} \sqrt{E_{p}+s M} J(n-\delta, n-l)\right. \\
& \left.+\sqrt{E_{q}-r M} \sqrt{E_{p}-s M} J(n+1-\delta, n-l)\right] \\
- & k_{3} R\left[\sqrt{E_{q}+r M} \sqrt{E_{p}-s M} J(n-\delta, n-l-1)\right. \\
- & \left.\sqrt{E_{q}-r M} \sqrt{E_{p}+s M} J(n+1-\delta, n-l+1)\right],
\end{aligned}
$$

so that

$$
\begin{aligned}
m_{\pi}= & \frac{4 e^{i(l-n) \varphi_{k}-i \frac{\pi}{2}|l-n|+i \pi n} \sin \pi \delta}{\sqrt{p_{\perp}^{4}-2 p_{\perp}^{2}\left(q_{\perp}^{2}+k_{\perp}^{2}\right)+\left(q_{\perp}^{2}-k_{\perp}^{2}\right)^{2}}}(a b)^{|n|} b^{|l|} \frac{1}{\omega_{k}} \\
\times & \left\{k _ { \perp } R _ { \perp } \left[\sqrt{E_{q}+r M} \sqrt{E_{p}+s M}\left(\Theta(l \geq 0) \Theta(n<0) a^{\delta}-\Theta(l<0) \Theta(n \geq 0) a^{-\delta}\right)\right.\right. \\
& \left.-\sqrt{E_{q}-r M} \sqrt{E_{p}-s M}\left(\Theta(l \geq 0) \Theta(n<0) a^{-1+\delta}-\Theta(l<0) \Theta(n \geq 0) a^{1-\delta}\right)\right] \\
- & k_{3} R\left[\sqrt{E_{q}+r M} \sqrt{E_{p}-s M}\left(\Theta(l \geq 0) \Theta(n<0) a^{\delta} b-\Theta(l<0) \Theta(n \geq 0) a^{-\delta} b^{-1}\right)\right. \\
& \left.\left.+\sqrt{E_{q}-r M} \sqrt{E_{p}+s M}\left(\Theta(l \geq 0) \Theta(n<0) a^{-1+\delta} b^{-1}-\Theta(l<0) \Theta(n \geq 0) a^{1-\delta} b\right)\right]\right\} .
\end{aligned}
$$

Eq. (30) together with (40) and (46) is the final expression for the bremsstrahlung matrix element with respect to cylindrical modes.

\subsection{Cross section for scattering states}

It is now very easy to calculate the matrix element of the bremsstrahlung process with respect to the electron scattering states (22). Consider an electron with momentum $\vec{p}$ and spin $s$ which is scattered by

the magnetic string and emits a photon with momentum $\vec{k}$ and polarization $\lambda$. Denoting the momentum and spin of the scattered electron $\vec{q}$ and $r$ resp. the corresponding matrix element reads

$$
\begin{aligned}
M_{\lambda} & :=-i\left\langle(\vec{q}, r),(\vec{k}, \lambda)\left|S^{(1)}\right|(\vec{p}, s)\right\rangle \\
& =\sum_{l, n} c_{l}^{(+)} c_{n}^{(-)^{*}} \widetilde{M}_{\lambda}\left(j_{p}, j_{q}\right) \\
& =\frac{-e \sqrt{2} \sin \pi \delta}{\sqrt{\omega_{k} E_{q} E_{p}}} \frac{\delta\left(E_{p}-E_{q}-\omega_{k}\right) \delta\left(p_{3}-q_{3}-k_{3}\right)}{\sqrt{p_{\perp}^{4}-2 p_{\perp}^{2}\left(q_{\perp}^{2}+k_{\perp}^{2}\right)+\left(q_{\perp}^{2}-k_{\perp}^{2}\right)^{2}}} \Sigma_{\lambda}
\end{aligned}
$$


where the coefficients $c_{l}^{(+)}$and $c_{n}^{(-)}$are given in eqs. (23) and (24) and refer to the ingoing $(\vec{p}, s)$ and outgoing electron $(\vec{q}, r)$ resp. It is

$$
\begin{aligned}
i \Sigma_{\sigma}:= & \sum_{l, n} c_{l, n}(a b)^{|n|} b^{|l|} R \\
& \times\left\{\sqrt{E_{q}+r M} \sqrt{E_{p}-s M}\left[\Theta(l \geq 0) \Theta(n<0) a^{\delta} b-\Theta(l<0) \Theta(n \geq 0) a^{-\delta} b^{-1}\right]\right. \\
& \left.-\sqrt{E_{q}-r M} \sqrt{E_{p}+s M}\left[\Theta(l \geq 0) \Theta(n<0) a^{-1+\delta} b^{-1}-\Theta(l<0) \Theta(n \geq 0) a^{1-\delta} b\right]\right\} \\
\Sigma_{\pi}:= & \sum_{l, n} c_{l, n}(a b)^{|n|} b^{|l|} \frac{1}{\omega_{k}} \\
& \times\left\{k _ { \perp } R _ { \perp } \left[\sqrt{E_{q}+r M} \sqrt{E_{p}+s M}\left(\Theta(l \geq 0) \Theta(n<0) a^{\delta}-\Theta(l<0) \Theta(n \geq 0) a^{-\delta}\right)\right.\right. \\
& \left.-\sqrt{E_{q}-r M} \sqrt{E_{p}-s M}\left(\Theta(l \geq 0) \Theta(n<0) a^{-1+\delta}-\Theta(l<0) \Theta(n \geq 0) a^{1-\delta}\right)\right] \\
& -k_{3} R\left[\sqrt{E_{q}+r M} \sqrt{E_{p}-s M}\left(\Theta(l \geq 0) \Theta(n<0) a^{\delta} b-\Theta(l<0) \Theta(n \geq 0) a^{-\delta} b^{-1}\right)\right. \\
& \left.\left.+\sqrt{E_{q}-r M} \sqrt{E_{p}+s M}\left(\Theta(l \geq 0) \Theta(n<0) a^{-1+\delta} b^{-1}-\Theta(l<0) \Theta(n \geq 0) a^{1-\delta} b\right)\right]\right\}
\end{aligned}
$$

with the new coefficients

$$
c_{l, n}:=e^{-i l\left(\varphi_{p}-\varphi_{k}\right)+i n\left(\varphi_{q}-\varphi_{k}\right)+i \frac{\pi}{2}\left(\epsilon_{l}+\epsilon_{n}\right) \delta} .
$$

After performing the sums over the angular quantum numbers $l, n$ and sorting with respect to the flux parameter $\delta$ we obtain the following closed expression.

$$
\Sigma_{\lambda}=\left(A_{\lambda} \Sigma_{+} a^{\delta}-B_{\lambda} \Sigma_{-} a^{-\delta}\right),
$$

with

$$
\begin{gathered}
\Sigma_{+}:=\frac{1}{1-a b e^{-i\left(\varphi_{p}-\varphi_{k}\right)}} \frac{b e^{-i\left(\varphi_{q}-\varphi_{k}\right)}}{1-b e^{-i\left(\varphi_{q}-\varphi_{k}\right)}}, \\
\Sigma_{-}:=\frac{a b e^{i\left(\varphi_{p}-\varphi_{k}\right)}}{1-a b e^{i\left(\varphi_{p}-\varphi_{k}\right)}} \frac{1}{1-b e^{i\left(\varphi_{q}-\varphi_{k}\right)}}, \\
A_{\sigma}:=\quad R\left[b \sqrt{E_{q}+r M} \sqrt{E_{p}-s M}-\frac{1}{a b} \sqrt{E_{q}-r M} \sqrt{E_{p}+s M}\right], \\
B_{\sigma}:=\quad R\left[\frac{1}{b} \sqrt{E_{q}+r M} \sqrt{E_{p}-s M}-a b \sqrt{E_{q}-r M} \sqrt{E_{p}+s M}\right], \\
A_{\pi}:=\frac{k_{\perp} R_{\perp}}{\omega_{k}}\left[\sqrt{E_{q}+r M} \sqrt{E_{p}+s M}-\frac{1}{a} \sqrt{E_{q}-r M} \sqrt{E_{p}-s M}\right] \\
B_{\pi}:=\frac{k_{\perp} R_{\perp}}{\omega_{k}}\left[\sqrt{E_{q}+r M} \sqrt{E_{p}+s M}-a \sqrt{E_{q}-r M} \sqrt{E_{p}-s M}\right] \\
\quad-\frac{k_{3}}{\omega_{k}} R\left[\frac{1}{b} \sqrt{E_{q}+r M} \sqrt{E_{p}-s M}+a b \sqrt{E_{q}-r M} \sqrt{E_{p}+s M}\right] .
\end{gathered}
$$

Eqn. (47) and (50) together with (51)-(55) contain the results for the bremsstrahlung matrix element with respect to scattering states.

Based on the matrix element (47) we evaluate the differential probability per unit length of string solenoid for the bremsstrahlung process:

$$
d W_{\lambda}=W_{\lambda} q_{\perp} d q_{\perp} d \varphi_{q} k_{\perp} d k_{\perp} d \varphi_{k} d q_{3} d k_{3},
$$


where

$$
W_{\lambda}:=\left|M_{\lambda}\right|^{2}=\frac{e^{2} \sin ^{2} \pi \delta}{8 \pi^{4} \omega_{k} E_{q} E_{p}} \cdot \delta\left(E_{p}-E_{q}-\omega_{k}\right) \delta\left(p_{3}-q_{3}-k_{3}\right) P_{\lambda}(\vec{q}, \vec{k})
$$

with

$$
P_{\lambda}(\vec{q}, \vec{k}):=\frac{\left|\Sigma_{\lambda}\right|^{2}}{p_{\perp}^{4}-2 p_{\perp}^{2}\left(q_{\perp}^{2}+k_{\perp}^{2}\right)+\left(q_{\perp}^{2}-k_{\perp}^{2}\right)^{2}} .
$$

In order to calculate the absolute value of (50) we shall make use of the fact that the Dirac equation (11) in the external Aharonov-Bohm field is covariant under boost transformation along the string direction. This means that it is sufficient to treat the case of normal incidence of the ingoing electron on the magnetic string and therefore we may perform all calculations in the coordinate system in which $p_{3}=0$. No information will be lost but the calculations become more simple in this case. For $p_{3}=0$ we use, instead of $q_{\perp}$ and $k_{\perp}$, more convenient variables: the dimensionless photon energy $\omega$ and the angle $\vartheta_{k}$ between the photon momentum and the string direction, defined by

$$
\omega:=\frac{\omega_{k}}{p_{\perp}}=\frac{p_{\perp}^{2}-q_{\perp}^{2}+k_{\perp}^{2}}{2 p_{\perp} \sqrt{p_{\perp}^{2}+M^{2}}}, \quad x:=\sin \vartheta_{k}=\frac{2 k_{\perp} \sqrt{p_{\perp}^{2}+M^{2}}}{p_{\perp}^{2}-q_{\perp}^{2}+k_{\perp}^{2}} .
$$

Referring to these variables we have $k_{\perp}=p_{\perp} \omega x, q_{\perp}=p_{\perp} \sqrt{1-2 \frac{\omega}{v}+\omega^{2} x^{2}}, v$ being the velocity of the ingoing electron, and

$$
a=\frac{1-\frac{\omega}{v}-\frac{\omega}{v} \sqrt{1-v^{2} x^{2}}}{\sqrt{1-2 \frac{\omega}{v}+\omega^{2} x^{2}}}, \quad b=\frac{1-\sqrt{1-v^{2} x^{2}}}{v x} .
$$

The variable $\omega$ ranges from 0 to $\omega_{\max }=v\left(1+\sqrt{1-v^{2} x^{2}}\right)^{-1}$ which corresponds to the minimal value of $q_{\perp}=0$. Calculating

$$
\begin{aligned}
\left|\Sigma_{+}\right|^{2}= & a \Sigma, \quad\left|\Sigma_{-}\right|^{2}=\frac{1}{a} \Sigma \\
\Sigma_{+} \Sigma_{-}^{*}+\Sigma_{+}^{*} \Sigma_{-}^{*}= & \frac{2}{a b^{2}} \Sigma^{2}\left\{\left[\left(1+b^{2}\right) \cos \varphi_{p k}-2 b\right]\left[\left(1+a^{2} b^{2}\right) \cos \varphi_{q k}-2 a b\right]\right. \\
& \left.-\left(b^{2}-1\right)\left(a^{2} b^{2}-1\right) \sin \varphi_{p k} \sin \varphi_{q k}\right\}
\end{aligned}
$$

with

$$
\Sigma:=\frac{a b^{2}}{\left(1+b^{2}-2 b \cos \varphi_{p k}\right)\left(1+a^{2} b^{2}-2 a b \cos \varphi_{q k}\right)}, \quad \varphi_{p k}:=\varphi_{p}-\varphi_{k}
$$

we obtain

$$
P_{\lambda}=\frac{P_{\lambda}^{(+)} a^{2 \delta}+P_{\lambda}^{(-)} a^{-2 \delta}+P_{\lambda}^{(0)}}{8 E_{p}^{2} \omega^{2}\left(1-v^{2} x^{2}\right)\left(1-v x \cos \varphi_{p k}\right)\left(1-\omega v x^{2}-v x \sqrt{1-2 \frac{\omega}{v}+\omega^{2} x^{2}} \cos \varphi_{q k}\right)},
$$

where, again, we sorted with respect to the dependence on the flux parameter $\delta$. We find for the polarization state $\sigma$

$$
\begin{aligned}
P_{\sigma}^{( \pm)}= & \frac{1}{2 r s}\left\{(r s+1)\left(1-v^{2} x^{2}\right)\left(2-2 \frac{\omega}{v}+\omega^{2} x^{2}\right)-\omega^{2}\left(1-x^{2}\right)\left(2-v^{2} x^{2}-\omega v x^{2}\right)\right. \\
& \left.\mp \sqrt{1-v^{2} x^{2}}\left(2-\omega v x^{2}\right)\left[(r+s) \frac{M \omega}{p_{\perp}}+s \frac{E_{p}}{M} \omega^{2}\left(1-x^{2}\right)\right]\right\}, \\
P_{\sigma}^{(0)}= & \frac{1}{r s} \frac{q_{\perp}}{p_{\perp}} F(\varphi)\left\{2(r s+1)\left(1-v^{2} x^{2}\right)+v^{2} \omega^{2} x^{2}\left(1-x^{2}\right)\right\},
\end{aligned}
$$

with

$$
F(\varphi):=\frac{\left(\cos \varphi_{p k}-v x\right)\left[p_{\perp}\left(1-\omega v x^{2}\right) \cos \varphi_{q k}-v x q_{\perp}\right]-p_{\perp}\left(1-v^{2} x^{2}\right) \sin \varphi_{p k} \sin \varphi_{q k}}{\left(1-v x \cos \varphi_{p k}\right)\left[p_{\perp}\left(1-\omega v x^{2}\right)-v x q_{\perp} \cos \varphi_{q k}\right]},
$$

and for the polarization state $\pi$

$$
P_{\pi}^{( \pm)}=\frac{1}{2 r s}\left\{(r s+1)\left[2\left(1-x^{2}\right)\left(1-\frac{\omega}{v}\right)+\omega^{2} x^{2}\left(1-v^{2} x^{2}\right)\right]\right.
$$




$$
\begin{aligned}
& \mp \sqrt{1-v^{2} x^{2}}\left[(r+s) \frac{M \omega}{p_{\perp}}\left(2-2 x^{2}-\omega v x^{2}\right)+s \frac{E_{p}}{M} \omega^{2}\left(1-x^{2}\right)\left(2-2 v^{2} x^{2}-\omega v x^{2}\right)\right. \\
& \left.\left.+s \frac{M}{p_{\perp}} 2 \omega^{2} v x^{4}\right]-\omega^{2}\left[\left(1+x^{2}\right)\left(1-v^{2} x^{2}\right)+\left(1-x^{2}\right)\left(1-\omega v x^{2}\right)\right]\right\}, \\
P_{\pi}^{(0)}= & -\frac{1}{r s} \frac{p_{\perp}}{q_{\perp}} F(\varphi)\left(1-x^{2}\right)\left\{2(r s+1)-\omega^{2} v^{2} x^{2}\right\} .
\end{aligned}
$$

The result for $P_{\lambda}$, and thus for the differential probability $d W_{\lambda}$ of eq. (57), is given by eq. (64) together with (65)-(69).

In real experiments one observes the momentum and polarization distributions of scattered electrons and bremsstrahlung photons. Complete information about this is contained in the differential radiativescattering cross section per unit length of the solenoid

$$
\frac{d \sigma_{\lambda}}{d \omega_{k} d \Omega_{k} d \varphi_{q}}=\left.\frac{e^{2} \sin ^{2} \pi \delta}{8 \pi^{4} p_{\perp}} \omega_{k} P_{\lambda}(\vec{q}, \vec{k})\right|_{E_{q}=E_{p}-\omega_{k}, q_{3}=p_{3}-k_{3}}
$$

whereby $d \Omega_{k}=d \cos \vartheta_{k} d \varphi_{k}$.

The formula (70) together with (64)-(69) contains the complete information about energy, angular and polarization distributions for the bremsstrahlung process. It allows to analyse correlations between energy and direction of the emitted radiation. We will now discuss the cross section at different regimes of the incoming electron's energy.

\section{The differential cross sections at different energies}

For the soft bremsstrahlung process with small photon energy the infrared singularity appears in the cross section in the limit $\omega \rightarrow 0$ as usual. This happens for any charged particle being scattered, and is not specific neither for the Dirac electron nor the Aharonov-Bohm field. The reason for this singularity is the failure of the perturbative expansion for soft photon emission.

For low electron energy $(v \ll 1)$ we have $\omega \leq \frac{v}{2}, a^{2} \approx 1-\frac{\omega_{k}}{E_{p}-M}$ and $s, r= \pm 1$. Then

$$
\begin{array}{cc}
P_{\sigma}^{( \pm)}=2 \Theta(s r)\left(1-\frac{\omega}{v} \mp s \frac{\omega}{v}\right), & P_{\pi}^{( \pm)}=\cos ^{2} \vartheta_{k} P_{\sigma}^{( \pm)}, \\
P_{\sigma}^{(0)}=2 \Theta(s r) a \cos \left(\varphi_{p k}+\varphi_{q k}\right), & P_{\pi}^{(0)}=-\cos ^{2} \vartheta_{k} P_{\sigma}^{(0)},
\end{array}
$$

and the differential cross section takes the form

$$
\frac{d \sigma_{\lambda}}{d \omega_{k} d \Omega_{k} d \varphi_{q}}=\frac{e^{2} \sin ^{2} \pi \delta}{32 \pi^{4} M \omega_{k}} v \Theta(s r) S^{(s)}\left(\begin{array}{c}
1 \\
\cos ^{2} \vartheta_{k}
\end{array}\right), \quad \text { for } \quad\left\{\begin{array}{l}
\lambda=\sigma \\
\lambda=\pi
\end{array}\right.
$$

with

$$
S^{(s)}:=\left(1-\frac{\omega_{k}}{E_{p}-M}\right)^{1+s \delta}+\left(1-\frac{\omega_{k}}{E_{p}-M}\right)^{-s \delta}+2 \epsilon_{\lambda}\left(1-\frac{\omega_{k}}{E_{p}-M}\right)^{\frac{1}{2}} \cos \left(\varphi_{p k}+\varphi_{q k}\right),
$$

where $\epsilon_{\lambda}=1$ for $\lambda=\sigma$ and $\epsilon_{\lambda}=-1$ for $\lambda=\pi$. The cross section of the bremsstrahlung process is rather small. It is proportional to the classical electron radius, $r_{0}=e^{2} / 4 \pi M$, and the velocity $v$ of the ingoing electron.

Let us note some particular features of the low energy bremsstrahlung process for the Dirac electron which can be read off from eq. (73).

(i) The electron spin projection is conserved at low electron energies as well as for soft photon emission at arbitrary energies.

(ii) The appearance of the additional factor $\cos ^{2} \vartheta_{k}$ for the photon polarization state $\pi$ is typical for the angular distribution of radiation from nonrelativistic particles. But the angular distributions of the outgoing electron and emitted photon are not independent on the angles $\varphi_{q}$ and $\varphi_{k}$ as it is the case for spinless particles. In addition to the first two terms, which appear for spinless particles too, for the 
Dirac electron an additional term occurs. This Dirac-specific term is dependent on the direction of the radiation relative to the momenta of the ingoing and outgoing electron. However, it does not depend on the field strength of the magnetic string and therefore it seems to be obvious that it arises due to the interaction of the electron's and the photon's spin.

(iii) After integration over the angle $\varphi_{q}$ of the outgoing electron only the first two terms remain and one finds

$$
S^{(s)}=2 \pi\left\{\left(1-\frac{\omega_{k}}{E_{p}-M}\right)^{1+s \delta}+\left(1-\frac{\omega_{k}}{E_{p}-M}\right)^{-s \delta}\right\} .
$$

The cross section then coincides for $s=-1$ with the one for nonrelativistic spinless particles [9]. In this case it is invariant under the transformation $\delta \rightarrow 1-\delta$, and consequently also under $\Phi \rightarrow-\Phi$. For $s=+1$, however, the interaction between the spin and the magnetic field leads to an attractive interaction between the spin and the magnetic field and results in a modification of the cross section. For spinless particles and for Dirac electrons with spin antiparallel to the magnetic string the wavefunctions vanish at the origin. Consequently their cross sections coincide at low energies. For an electron with antiparallel spin projection, $s=+1$ in our case, the wave function is amplified near the string and this leads to a different cross section.

(iv) The photon emission with electron spin-flip takes place for higher electron energies. As a result of the scattering process the electron tends to direct its spin antiparallel to the magnetic field. It leads to a small self-polarization effect for the electron beam in the same manner as it happens for synchrotron radiation 18 .

We now discuss the angular distribution in the case of high electron energy, when $\gamma=1 / \sqrt{1-v^{2}} \gg 1$. Due to the presence of the factors

$$
\left(1-v x \cos \varphi_{p k}\right) \sim E_{p} \omega_{k}-\vec{p} \vec{k}
$$

and

$$
\left(1-\omega v x^{2}-v x \sqrt{1-2 \frac{\omega}{v}+\omega^{2} x^{2}} \cos \varphi_{q k}\right) \sim E_{q} \omega_{k}-\vec{q} \vec{k}
$$

in the denominator of (64) the bremsstrahlung cross section has a sharp maximum in the neighborhood of the direction of the ingoing electron, and the radiation is concentrated within a narrow cone around this direction. This cone has an angular aperture of order of magnitude $\sim 1 / \gamma$. The same cone also contains the momentum of the scattered electron. Outside the probability of radiative transition decreases rapidly.

\section{The total cross section}

Let us now integrate over final states in order to find the total cross section. After integrating over the angle $\varphi_{q}$ of the outgoing electron the term with $P^{(0)}$ disappears and we obtain

$$
\frac{d \sigma_{\lambda}}{d \omega_{k} d \Omega_{k}}=\frac{e^{2} \sin ^{2} \pi \delta}{32 \pi^{3} \omega_{k}} \frac{v}{E_{p}} \frac{P_{\lambda}^{(+)} a^{2 \delta}+P_{\lambda}^{(-)} a^{-2 \delta}}{\left(1-v^{2} x^{2}\right)^{\frac{3}{2}}\left(1-v x \cos \varphi_{p k}\right)} .
$$

Summing over polarization states of the outgoing electron (indicated by a bar) we find

$$
\frac{d \bar{\sigma}_{\lambda}}{d \omega_{k} d \Omega_{k}}=\frac{e^{2} \sin ^{2} \pi \delta}{32 \pi^{3} \omega_{k}} \frac{v}{E_{p}} \frac{\bar{P}_{\lambda}^{(+)} a^{2 \delta}+\bar{P}_{\lambda}^{(-)} a^{-2 \delta}}{\left(1-v^{2} x^{2}\right)^{\frac{3}{2}}\left(1-v x \cos \varphi_{p k}\right)}
$$

with

$$
\bar{P}_{\sigma}^{( \pm)}=\left(1-v^{2} x^{2}\right)\left(2-2 \frac{\omega}{v}+\omega^{2} x^{2}\right) \mp s \sqrt{1-v^{2} x^{2}}\left(2-\omega v x^{2}\right) \frac{M \omega}{p_{\perp}},
$$

and

$$
\bar{P}_{\pi}^{( \pm)}=2\left(1-x^{2}\right)\left(1-\frac{\omega}{v}\right)+\omega^{2} x^{2}\left(1-v^{2} x^{2}\right) \mp s \sqrt{1-v^{2} x^{2}}\left(2-2 x^{2}-\omega v x^{2}\right) \frac{M \omega}{p_{\perp}} .
$$

The integration over the angle $\varphi_{k}$ of the emitted photon leads to an additional factor $2 \pi\left(1-v^{2} x^{2}\right)^{-\frac{1}{2}}$. We find as result

$$
\frac{d \bar{\sigma}_{\lambda}}{d \omega_{k} d \cos \vartheta_{k}}=\frac{e^{2} \sin ^{2} \pi \delta}{16 \pi^{2} \omega_{k}} \frac{v}{E_{p}} \frac{\bar{P}_{\lambda}^{(+)} a^{2 \delta}+\bar{P}_{\lambda}^{(-)} a^{-2 \delta}}{\left(1-v^{2} x^{2}\right)^{2}}
$$


The remaining integrals over $d \omega_{k}$ and $d \vartheta_{k}$ can not be found analytically for arbitrary electron energies. For low electron energies we have

$$
\frac{d \bar{\sigma}_{\sigma}}{d \omega_{k}}=3 \frac{d \bar{\sigma}_{\pi}}{d \omega_{k}}=\frac{r_{0} \sin ^{2} \pi \delta}{\pi} \frac{v}{\omega_{k}}\left[\left(1-\frac{\omega_{k}}{E_{p}-M}\right)^{1+s \delta}+\left(1-\frac{\omega_{k}}{E_{p}-M}\right)^{-s \delta}\right] .
$$

This result can also be obtained from (73) by integrating over $\varphi_{q}, \varphi_{k}$ and $\vartheta_{k}$, i. e. by first making the approximation of low energy and summing over final states afterwards.

For high electron energies, $\gamma=\left(1-v^{2}\right)^{-\frac{1}{2}} \gg 1$, the main contribution to the cross section arises from values of $\vartheta_{k} \sim \frac{\pi}{2}$, or $x \sim 1$. In this case $a \approx 1$ if the bremsstrahlung photon is not too hard, $\omega<\omega_{\max }$, and we obtain for the cross section

$$
\frac{d \bar{\sigma}_{\lambda}}{d \omega_{k}} \sim \frac{r_{0} \sin ^{2} \pi \delta}{\omega_{k}} f_{\lambda}\left(\omega_{k}\right)
$$

where $f_{\lambda}\left(\omega_{k}\right)$ is a function of the photon energy. It means that the form of the energy bremsstrahlung spectrum is independent on the ingoing electron energy and polarization state. For hard photons, $\omega \sim \omega_{\max }$, we have $a^{2} \sim\left(\omega_{\max }-\omega\right) / 2 \sqrt{1-v^{2} x^{2}}$, and the cross section behaves asymptotically as

$$
\frac{d \bar{\sigma}_{\lambda}}{d \omega_{k}} \sim \frac{r_{0} \sin ^{2} \pi \delta}{\omega_{k}} \gamma^{\delta}\left(1-\frac{\omega_{k}}{E_{p}}\right)^{\delta}(a \mp b s),
$$

where $a$ and $b$ are coefficients and the signs of $\mp$ correspond to $\lambda=\sigma$ and $\pi$ resp. It means that the fraction of hard photons increases with the ingoing electron energy. i.e. the bremsstrahlung photon spectrum becomes more hard. Also there is a correlation between the electron spin states and the photon polarization.

\section{Conclusions}

We have analyzed the bremsstrahlung of an electron being scattered by a magnetic string, which, among other quantum processes accompanying the $\mathrm{AB}$ scattering, seems to be the most interesting and significant one.

In addition to the $\mathrm{AB}$ interaction, resulting from the non-integrable phase factors, which all quantum particles suffer, spin particles interact with the magnetic field via their magnetic moments. This strongly influences their behaviour near the flux tube. In the idealized case of an infinitely thin magnetic string their wave functions do not vanish on the string and the non-locality of the $\mathrm{AB}$ effect is modified by a local interaction. This interaction leads to a specific behaviour of the cross section.

We evaluated the differential radiative cross section, which contains complete information about energy, angular and polarization distribution of the participating particles, as well as the total cross section and analysed them for different energy regimes. For low electron energies an angular asymmetry in the plane perpendicular to the string occurs. This result may be the most interesting one for possible experimental observations.

The bremsstrahlung allows for attempts of detailed experimental investigation of the AB effect. Of course, the observation of the $\mathrm{AB}$ effect, which is done by means of electron interference and electron holography [6], is not a simple task and the radiative $\mathrm{AB}$ effect for polarized electron beams requires a careful discussion.

We draw attention to a remarkable feature which is characteristic for quantum processes in the presence of the $\mathrm{AB}$ string both for spinless and for spin particles. The processes happen if the ingoing and outgoing electrons have angular momentum projection of opposite signs. In a sense the electron needs to circle the $\mathrm{AB}$ string to emit the bremsstrahlung photon. In this case it can transmit a part of its perpendicular momentum to the string. The relative excess, $\Delta=1-\left(q_{\perp}+k_{\perp}\right) / p_{\perp}$, is ranged from its minimal value $\Delta=0$ at $\omega_{k}=0$ to the maximal value $\Delta=1$ at $\omega_{k}=p_{\perp} v / 2$ when the bremsstrahlung photon takes away all kinetic energy of the electron.

Finally we point out the analogy to the physics of an electron passing a cosmic string [19]. In this case an additional term appears which results from the spin connection. It corresponds to the vector potential term in the $\mathrm{AB}$ case. 


\section{Acknowledgments}

V. S. thanks J. Audretsch and the members of his group at the University of Konstanz for hospitality, collaboration and many fruitful discussions. This work was supported by the Deutsche Forschungsgemeinschaft.

\section{References}

[1] Y. Aharonov and D. Bohm, Phys. Rev. 119, 485 (1959).

[2] W. Franz, Verh. Deutsch. Physik. Ges. 20, 65 (1939).

[3] W. Ehrenberg and R. E. Siday, Proc. Phys. Soc. London B 62, 8 (1949).

[4] T. T. Wu and C. N. Yang, Phys. Rev. D 12, 3864 (1975).

[5] S. Olariu and I. I. Popescu, Rev. Mod. Phys. 47, 339 (1985).

[6] M. Peshkin and A. Tonomura, The Aharonov-Bohm effect, Springer-Verlag, Berlin (1989).

[7] P. A. Lee and T. V. Ramakrishnan, Rev. Mod. Phys. 57, 287 (1985).

[8] G. Bergmann, Phys. Rep. 107, 1 (1984).

[9] E. M. Serebryanyı̆, Theor. Math. Phys. 64, 846 (1986).

[10] E. M. Serebryanyı̆ and V. D. Skarzhinskiǔ, Sov. Phys. - Leb. Inst. Rep. 6, 56 (1988), and in Proc. P. N. Lebedev Phys. Inst. 197 (in Russian), ed. by M. A. Markov, Nauka, Moscow (1989)

[11] D. V. Gal'tsov and S. A. Voropaev, Sov. J. Nucl. Phys. 51, 1811 (1990).

[12] C. R. Hagen, Phys. Rev. Lett. 64, 503 (1990).

[13] C. R. Hagen, Int. J. Mod. Phys. A 6, 3119 (1991).

[14] Ph. S. de Gerbert, Phys. Rev. D 40, 1346 (1989).

[15] J. Audretsch, U. Jasper and V. D. Skarzhinsky, A pragmatic approach to the problem of the selfadjoint extension of Hamilton operators with the Aharonov-Bohm potential, to appear in J. Phys. A: Math. Gen. (1995)

[16] M. Reed and B. Simon, Methods of Modern Mathematical Physics vol. II, Fourier Analysis, SelfAdjointness, Academic Press, New York (1975).

[17] I. S. Gradshteyn and I. M. Ryzhik, Table of integrals, series and products, Academic Press (1980).

[18] A. A. Sokolov and I. M. Ternov, Synchrotron radiation, Akademie-Verlag, Berlin (1968).

[19] J. Audretsch, U. Jasper, and V. Skarzhinsky, Phys. Rev. D 49, 6576 (1994).

[20] C. R. Hagen and D. K. Park, Relativistic Aharonov-Bohm-Coulomb problem, hep-th/9410225 (1994). 